\title{
Epstein-Barr Virus Infection to Epstein-Barr Virus-Negative Nasopharyngeal Carcinoma Cell Line TW03 Enhances Its Tumorigenicity
}

\author{
Norihiro Teramoto, Akihiko Maeda, Keita Kobayashi, Kazuhiko Hayashi, \\ Takashi Oka, Kiyoshi Takahashi, Kenzo Takada, Georg Klein, and Tadaatsu Akagi \\ Department of Pathology (NT, KK, KH, TO, KTakah, TA), Okayama University Medical School, Okayama, Japan; \\ Microbiology and Tumor Biology Center (AM, GK), Karolinska Institute, Stockholm, Sweden; Department of Virology \\ (KTakad), Cancer Institute, Hokkaido University School of Medicine, Sapporo, Japan
}

\begin{abstract}
SUMMARY: Almost all nasopharyngeal carcinomas (NPCs) are infected by Epstein-Barr virus (EBV), but most ex vivo NPC cells lose EBV genomes during passages. In this study, an EBV-negative NPC cell line, TW03, established from EBV-carrying NPC was reinfected with EBV by cocultivation with irradiated Akata cells carrying recombinant EBV containing a neomycin-resistant gene. The reinfected EBV (+) TW03 cells expressed EBERs and EBNA1, but not EBNA2, lytic proteins (ZEBRA and EA-D), or LMP1. They had an epithelial appearance similar to that of EBV $(-)$ TW03 cells. The doubling times of EBV $(+)$ and EBV $(-)$ TW03 cells were almost identical. However, the EBV $(+)$ TW03 cells formed larger colonies with ragged contours in anchorage-independent cultures. An in vitro invasion assay showed that EBV $(+)$ TW03 cells had a higher invasive activity than EBV $(-)$ TW03 cells $(p<$ 0.01). Both EBV $(-)$ and EBV $(+)$ TW03 cells formed poorly differentiated squamous cell carcinomas in SCID and nude mice. EBV $(+)$ TW03 cells showed a higher tumorigenicity to nude mice $(12$ of 13$)$ than EBV $(-)$ TW03 cells $(1$ of 9$)(p<0.001)$. In the severe combined immunodeficiency (SCID) tumors of EBV $(+)$ TW03 cells, not all of the tumor cells were EBER-1 positive. EBER-1 was more frequently detected in the peripheral regions and daughter nodules of the tumors than in the central areas. The microdissection polymerase chain reaction showed that the EBER-1-negative TW03 cells in the EBV (+) TW03 SCID tumors lost EBV genomes. EBER-1-negative cells showed as high a rate of Ki-67 positivity as EBER-1-positive cells, indicating that the former were proliferating rather than dead or dying. In horny pearls, keratinizing cells were ZEBRA-positive and EBER-negative. Loss of EBV genomes was not associated with squamous differentiation. These data indicated that reinfection of EBV promotes the tumorigenicity of EBV (-) TW03 cells by enhancing the invading activity. (Lab Invest 2000, 80:303-312).
\end{abstract}

$E$ pstein-Barr virus (EBV) is a ubiquitous virus estimated to have infected more than $90 \%$ of human beings and known to have the strongest transforming ability among the human viruses (Klein, 1994). EBV is associated with a wide spectrum of human diseases, including most cases of nasopharyngeal carcinoma (NPC) and some cases of gastric cancer, endemic Burkitt lymphoma, immunoblastoma, and leiomyosarcoma of young immunocompromised hosts (Rickinson and Kieff, 1996).

NPC is the EBV-associated epithelial tumor most intensively studied. It is the most frequent carcinoma in South China (Raab, 1992). Almost all NPC tumor cells contain EBV. However, the etiological role of EBV in NPC remains to be clarified. A proportion of NPC is characterized by expression of latent membrane protein 1 (LMP1), in addition to expression of EBVencoded small RNAs (EBERs) and EBV-associated nuclear antigen-1 (EBNA1), both of which are always

Received October 5, 1999.

Address reprint requests to: Dr. N. Teramoto, Department of Pathology, Okayama University Medical School, Shikata-cho, 2-5-1, Okayama city, Okayama prefecture, 700-8558, Japan. Fax: 8186235 7156; E-mail: terra@med.okayama-u.ac.jp expressed in latently infected cells. This pattern of EBV infection is known as latency II (Rowe et al, 1992). LMP1 protein is detected only in one-half of NPC cases by Western blotting and in $10 \%$ to $20 \%$ by immunohistostaining. LMP1 is a very competent viral oncogene that can transform rodent fibroblasts (Wang et al, 1985). However, it is also an immunogenic protein that can be a target of rejection in vivo (Trivedi et al, 1991). EBNA1 (Roth et al, 1994; Sheu et al, 1996) and EBERs (Komano et al, 1998), which are not targeted by cytotoxic $T$ cells, have also been implicated in the tumorigenic activity of EBV. Interestingly, most NPC cells gradually lose EBV during passages in vitro (Lin et al, 1993), indicating that EBV does not have an advantage for cell proliferation in vitro.

EBV can immortalize resting B cells very efficiently in vitro (Kieff, 1996; Klein, 1994), but rarely infects epithelial cells in vitro. In a study by Nishikawa et al (1999), however, EBV infection in vitro conferred a transformed phenotype, promoting cellular growth in non-neoplastic gastric epithelial cells with prolonged survival.

There have been several reports that EBV can infect established non-B cell lines. Lin et al (1997) reported that NPC cells could uptake EBV particles through 
incorporation of secretary components if EBV particles were treated with Ig A anti-EBV viral capsid antigen (VCA). Yoshiyama et al (1997) reported that gastric carcinoma cell lines could be infected with the recombinant EBV with a selectable marker and Imai et al (1998) reported that the recombinant EBV could be transferred to epithelial cells more efficiently by cocultivation with the lytic EBV-positive Akata cells than cell-free supernatant containing the virus. However, the mechanism of EBV infection of epithelial cells remains uncertain. Recently, Holmgren et al (1999) reported that EBV can be transferred through uptake of apoptotic bodies (apoptotic body transfer), although in their study the transfer of the EBV genome was limited to the EBV DNA integrated into chromosomes.

In this study, an EBV-negative NPC cell line, TW03, was reinfected with EBV. TW03 is a cell line derived from an undifferentiated NPC with massive lymphocytic infiltration, which originally harbored EBV but lost it during successive passages (Lin et al, 1993). We report changes of the biological properties of this cell line caused by EBV reinfection.

\section{Results}

\section{EBV Infection of TW03 Cells}

Most TW03 cells died under the selection with G418 after cocultivation with neo-Akata cells. EBVreinfected TW03 colonies emerged 4 to 5 weeks later.
EBV (+) TW03 cells showed an epithelial pattern similar to that of the parental TW03 cells (Fig. 1, a to c). The doubling time of both EBV $(-)$ and EBV $(+)$ TW03 cells was found to be approximately 13 to 14 hours in $10 \%$ fetal calf serum/Iscove's medium (FCS/IMDM). The EBV ( - ) and EBV (+) TW03 cells proliferated almost equally in both standard (10\% FCS) and serum-deprived ( $0 \%$ or $2 \%$ FCS) culture media (Fig. 2). Most cells of the EBV-infected cultures were EBER-1 positive, whereas all parental TW03 cells were negative for EBER-1 (Fig. 1, $d$ and e). EBER-1negative cells emerged when the EBV-infected cells were cultured without G418 (Fig. 1f). A GC-contentmatched control probe for EBER-1 was totally unreactive (data not shown).

Human monospecific sera against EBNA1 reacted with almost all of the nuclei of EBV $(+)$ TW03 cells but not with the parental TW03 cells (Fig. 1, g to i). EBNA2, LMP1, ZEBRA, and EA-D were not detected in either EBV (-) or EBV (+) TW03 cells by indirect immunofluorescent staining (data not shown). A BamHIW fragment of EBV DNA was amplified from the EBV $(+)$ TW03 cells but not from the parental TW03 cells (Fig. 3a). RT-PCR detected expression of mRNA QUK but not $Y U K$, indicating that the $Q$ promoter was active whereas the $\mathrm{C}$ and $\mathrm{W}$ promoters were inactive (Fig. $3 b)$. Southern blot hybridization with the probe $1.9 \mathrm{~kb}$ Xhol fragment of EBV DNA detected EBV in EBV (+) TW03 cells was polyclonal (Fig. 3c). This indicated that the EBV $(+)$ TW03 cells were not derived from a
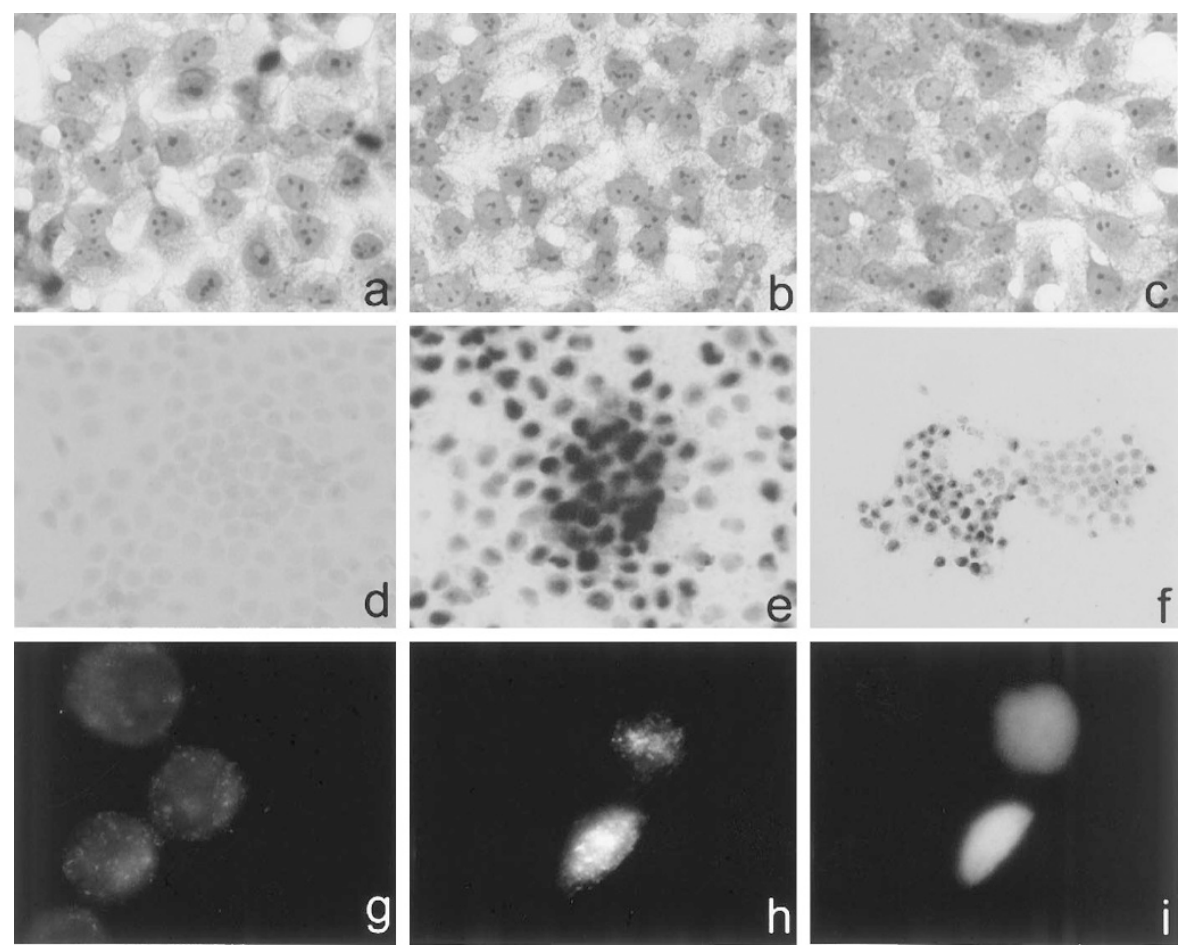

Figure 1.

TW03 and Epstein-Barr virus (EBV)-reinfected TW03 cells in vitro. a, $d$, and $g$ : EBV (-) parental TW03; $b, e, h$, and $i$ : EBV-reinfected TW03; $c$ and $f$. EBV reinfected TW03 cultured without G418 ( $c, 1$ month; $f, 1$ week). a to $c$ : May-Grünwald Giemsa stain. $d$ to $f$. EBER-1 in situ hybridization counterstained with Methylgreen. $g$ and $h$ : Anti-complement indirect immunofluorescence staining for EBNA1. $i$ : Nuclear staining by Hoechst. $h$ and $i$ show the same field. Original magnification: $\times 400$ (a to $c), \times 200(d, e), \times 100(f), \times 1000(g$ to $l)$. 


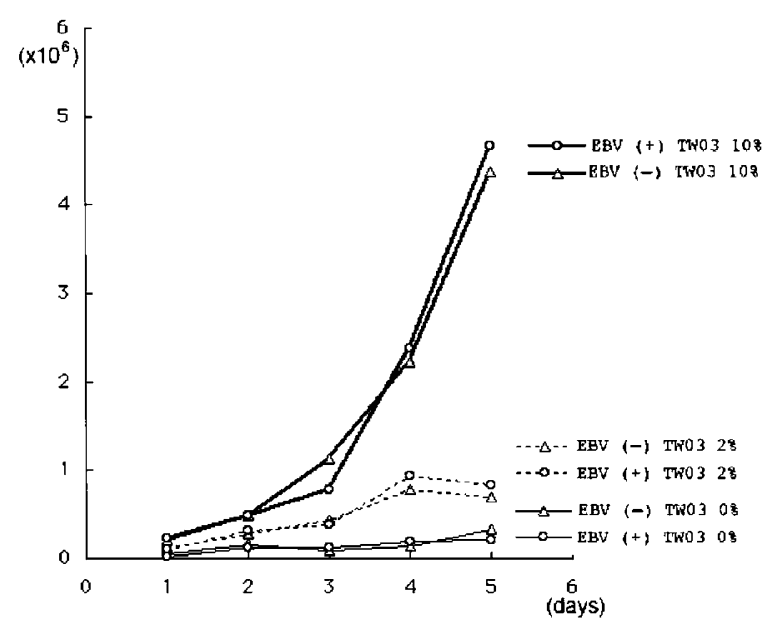

Figure 2.

In vitro proliferation of EBV $(+)$ and EBV (-) TW03 cells. 10\%,10\% fetal calf serum (FCS) $/ 90 \%$ Iscove's medium (IMDM); $2 \%, 2 \%$ FCS $/ 18 \%$ IMDM $/ 80 \%$ phosphate buffered saline (PBS); $0 \%, 20 \%$ IMDM/ $80 \%$ PBS.

particular subclone of TW03 cells. EBV DNA was not detected in the parental TW03 cells.

\section{Anchorage-Independent Cell Growth}

The cloning efficiency of the EBV $(-)$ and EBV (+) TW03 cells in semi-solid agarose culture was not significantly different (EBV (-) TW03: efficiency, 36\% to $54 \%$; mean, $49 \% \pm 7.4 \%$; EBV (+) TW03, efficiency, $35-54 \%$; mean, $45.8 \% \pm 7.6 \%)$. However, colonies larger than $1 \mathrm{~mm}$ in diameter were found only in the EBV (+) TW03 cultures. In the Sigmacotetreated chambers, the morphology of each colony was more clearly observed by hematoxylin eosin (HE) staining than by phase contrast in semi-solid agarose culture (Fig. 4). The size of EBV (+) TW03 colonies (mean, 442 pixels) was significantly larger than that of EBV (-) TW03 colonies (mean, 217 pixels) $(p<0.001$, double-sided $t$ test). The edges of the EBV (+) TW03 clusters were ragged (Fig. 4d), suggesting a higher potential of invasiveness, whereas EBV (-) TW03 clusters showed a smooth spheroid pattern (Fig. 4b).

\section{EBV-Induced Promotion of the Invasive Ability of TW03 Cells}

More EBV (+) TW03 cells passed through the pores and the growth factor reduced (GFR) Matrigel than EBV (-) TW03 cells although a proportion of EBV (-) TW03 cells also invaded the Matrigel (Fig. 5, a to d). As shown in a preliminary study (Fig. 5 e), the difference of the invasiveness seemed to be significant in the presence of $0.5 \mathrm{ng} / \mathrm{ml}$ of epidermal growth factor (EGF). Therefore, the percent of invasion at $0.5 \mathrm{ng} / \mathrm{ml}$ of EGF was examined 4 times. The percent of invasion of EBV (-) TW03 was $26.9 \%$ to $52.5 \%$ with a mean of $39 \% \pm$ $10.5 \%$ and that of EBV $(+)$ TW03 was $61.1 \%$ to $93.3 \%$ with a mean of $78.8 \% \pm 13.3 \%(p<0.01$, doublesided $t$ test) (Fig. 5f), and that of nontransformed mouse $L$ cells was quite low (mean $1.1 \% \pm 1.2 \%$ ).
Thus EBV infection markedly promoted the invasiveness of TW03 in vitro.

\section{Tumorigenicity of TW03 Cells into Nude Mice and SCID Mice}

Tumors were produced in 12 of the 13 nude mice injected with $5 \times 10^{6}$ EBV $(+)$ TW03 cells in 1 month, whereas EBV $(-)$ TW03 cells formed a tumor in only 1 of the 9 mice in 2 months ( $p<0.001, \chi^{2}$ test). No tumor was formed by injection of $5 \times 10^{5}$ or $5 \times 10^{4}$ EBV (+) TW03 cells or EBV (-) TW03 cells in nude mice. EBV (-) and EBV (+) TW03 cells produced tumors in almost all SCID mice in 2 months (4 of 5 and 9 of 9, respectively). Histologically, both the SCID and nude mice tumors were found to be poorly differentiated squamous cell carcinoma with minimal keratinization (Fig. 6, a and b). The EBV (-) and EBV (+) TW03 tumors were morphologically indistinguishable.

EBER-1 in situ hybridization (EBERISH) detected EBER-1 in most of the living cells of the EBV (+) TW03-induced nude mice tumors (Fig. 6c). Simultaneous staining for EBER-1 and cytokeratin showed that EBER-1 positive cells were epithelial cells. A GC-content-matched control probe was totally unreactive (Fig. 6c, insert). EBV (+) TW03-induced SCID tumors also contained EBERI-1 positive cells (Fig. 7).

In a few cases, horny pearls were observed (Fig. 6d). Horny pearls, which are keratinizing centers of squamous cell carcinoma, EBER-1, turned negative (Fig. $6 e)$, and ZEBRA was switched on to be expressed (Fig. 6f). This indicated that the EBV-infected state persisted during the differentiation from undifferentiated cells to keratinized cells and that keratinization was inconsistent with latent infection. Except within horny pearls, tumor cells were very rarely positive for ZEBRA (Fig. 6g) and were negative for LMP1 (Fig. 6 h). The tumor cells of the EBV (-) TW03-induced tumor were negative for EBER-1 and EB viral proteins.

\section{EBV is Preserved in the Infiltrating Borders and Daughter Nodules of SCID Mouse Tumors}

The daughter tumor nests of the EBV (+) TW03induced SCID mouse tumors contained more EBER1-positive cells than the main tumor masses (Fig. 7). EBER-1-positive cells were more frequently found in the periphery of the tumors and less often in the center of the tumors (Fig. 7b). Ki-67-positive cells were rather evenly distributed, and simultaneous staining for both Ki-67 and EBER-1 showed that EBER-1 negative cells were not dead or dying but actively proliferating (Fig. 7c). In situ hybridization for histone mRNA showed that RNA was rather evenly preserved even in the center of the tumor. PCR of the EBER-1-negative regions microdissected from the SCID tumor specimens did not amplify the EBV DNA (Figs. 7d and 8). Thus, EBER negativity was not due to the artificial RNA loss or reduced expression of EBERs of EBVcarrying cells, but rather was due to the loss of EBV genomes. The loss of EBV was not related to squamous differentiation. 
a

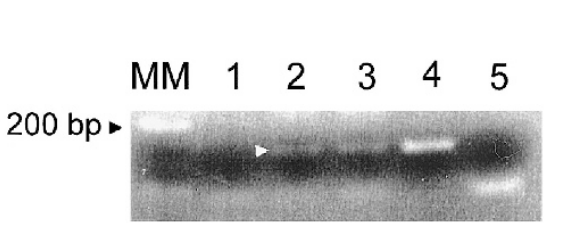

b

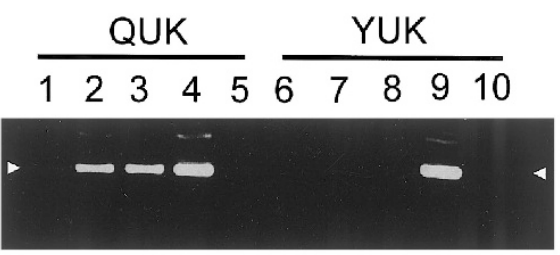

\section{C}

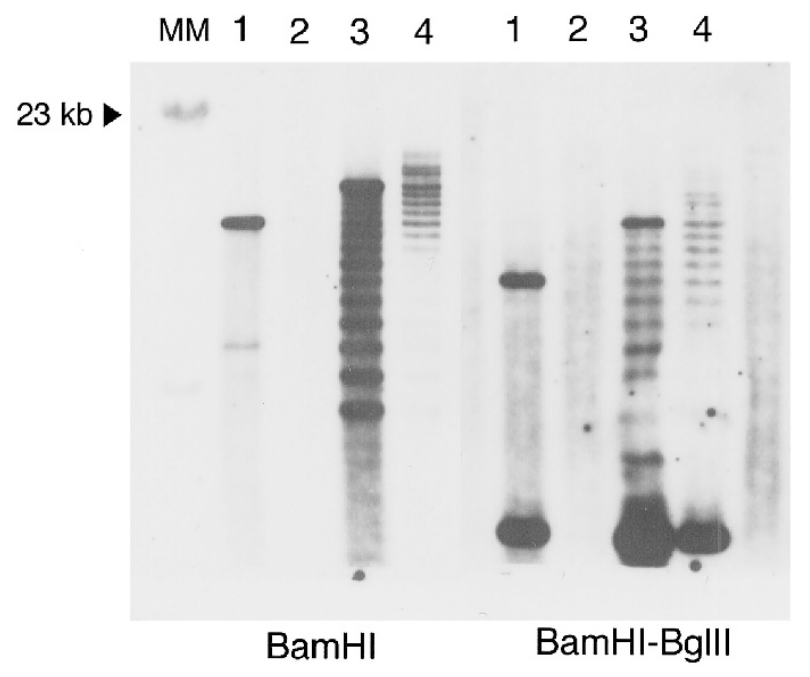

Figure 3.

Detection of EBV DNA and RNA. a, Polymerase chain reaction (PCR) of a BamHIW fragment of EBV DNA (125 bp) amplified from EBV (+) TW03. Lane 1, parental TW03; lanes 2 and 3, EBV (+) TW03; lane 4, neo-Akata; lane 5, distilled water. b, RT-PCR for QUK from Q promoter and YUK from C/W promoter. Lane 1, parental TW03; lanes 2 and 3, EBV (+) TW03; lane 4, neo-Akata; lane 5, distilled water; lane 6, parental TW03; lanes 7 and 8, EBV (+) TW03; lane 9, LCL; lane 10, distilled water. $c$, Southern hybridization for terminal repeats of EBV. Lane 1, LAD, (LCL); lane 2, parental TW03; lane 3, neo-Akata; lane 4, EBV (+) TW03. The single band in lane 1 shows that LAD cells were monoclonally infected with EBV, whereas the ladders in lane 4 indicate that EBV $(+)$ TW03 cells are polyclonally infected with EBV.

\section{Discussion}

Imai et al (1998) reported that various human epithelial cells can be efficiently infected with EBV through cell-to-cell contact with recombinant EBV-producing neo-Akata cells. In the present study, an EBV-negative NPC line, TW03, could be infected with EBV by simple co-cultivation with irradiated neo-Akata cells, a method originally reported by Holmgren et al (1999). In their report, Holmgren et al (1999) showed that EBV infected non-B cells through the transfer of apoptotic bodies. However, only the EBV genomes integrated into chromosomes were transferred to other cell nuclei. In the present study, we showed that episomal EBV could infect non-B cells using the same method together with selection by G418. However, it was unclear whether this EBV infection occurred via the transfer of apoptotic bodies or through an unknown receptor, because neo-Akata cells may have a small number of spontaneous replicated virions.

As shown in Figure 2, EBV infection did not promote TW03 proliferation in the adherent cultures at high or low concentrations of FCS. This is in line with the result that EBV was lost when the cells were cultured without G418 and the previous reports that most NPC cell lines lost EBV during passages (Lin et al, 1993; 1997). However, EBV (+) TW03 cells formed larger colonies in the cell suspension culture in the Sigmacote-treated chambers (Sigma, St. Louis, Missouri) and in soft agarose than EBV (-) TW03. The morphology of the cells in the colonies suggested that EBV (+) TW03 cells had the higher invasion potential. The invasion assay indicated that EBV infection promoted the invasion activity of TW03 cells in vitro. EBV (+) TW03 cells showed a higher tumorigenicity to nude mice than EBV (-) TW03 cells. According to the immunostaining results, this higher tumorigenicity was not due to the expression of LMP1.

EBERISH of the SCID tumor clearly showed that EBV infection was more frequently preserved in the cells of daughter tumor nodules and the periphery of the tumor masses than in the cells of the center of the main tumors (Fig. 7). Simultaneous staining for Ki-67 and EBER-1 showed that both EBV-positive and EBVnegative cells had proliferated as well. Loss of EBV neither abolished the proliferating activity nor promoted the cell death of the tumor cells because a 


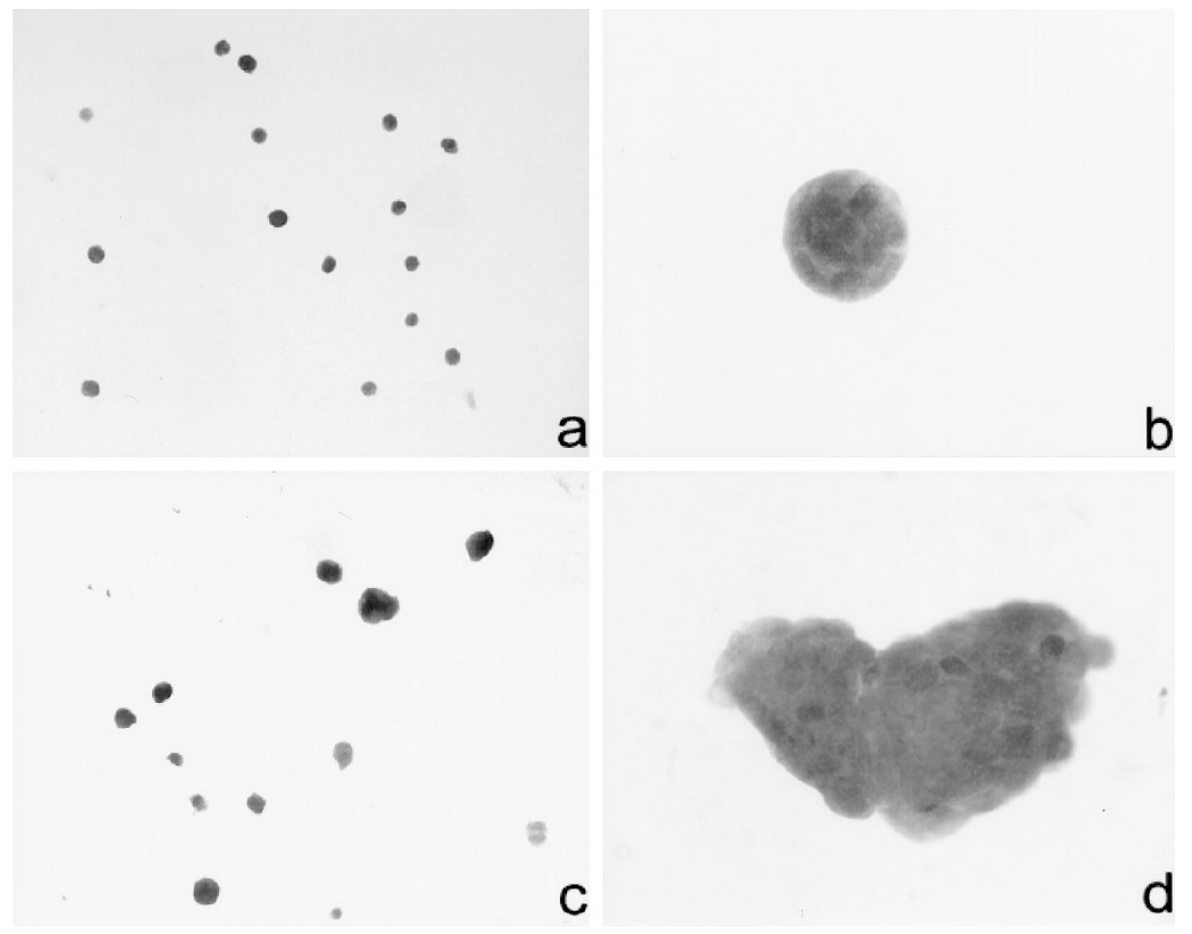

\section{Figure 4.}

Anchorage-independent culture of EBV (-) and EBV (+) TW03 cells in the Sigmacote-treated chambers. $a, b$ : EBV $(-)$ TW03. $c$, d: EBV (+) TW03. Hematoxylin eosin (HE) staining. Original magnification: $\times 25(a$ and $c), \times 400(b$ and $d)$.

substantial proportion of proliferating cells lost EBV in some tumors. EBV-carrying cells showed a greater invasiveness into the surrounding tissues than EBVlost cells. This finding was consistent with the data of the in vitro invasion assay and may explain the significant difference of tumorigenicity to nude mice between EBV (-) and (+) TW03 cells. EBV usually monoclonally infects NPC. Therefore, EBV is supposed to play an important role in NPC etiology at the early stage. The higher invading activity of EBV $(+)$ TW03 cells indicates that EBV might promote tumor progression of NPC from carcinoma in situ to invasive carcinoma.

Horny pearls were observed in the EBV (+) TW03induced mouse tumors. There EBV turned to the lytic phase of infection. It is well known that EBV infection is lytic in oral hairy leukoplakia and in keratinizing cells in nasopharyngeal carcinoma (Niedobitek et al, 1991; Pathmanathan et al, 1995). However, there is no good experimental model for analyzing the relations between EBV and keratinizing differentiation. This cell line might serve as such a model. In conclusion, EBV did not influence the morphology or cell proliferation of TW03 cells in adherent culture, whereas EBV promoted the tumorigenicity of TW03 cells, which may have been due to the invasive ability of EBV (+) TW03 cells.

\section{Materials and Methods}

\section{Cell Lines}

An NPC cell line, TW03, was kindly provided by Dr. C. T. Lin, Taipei, Taiwan, Republic of China (Lin et al,
1993). It was cultured in modified IMDM supplemented with 10\% FCS. EBV-negative Akata cells reinfected with the recombinant EBV containing a neomycin-resistant gene (neo-Akata) were cultured in RPMI1640 supplemented with 10\% FCS and 500 $\mu \mathrm{g} / \mathrm{ml}$ G418 (Shimizu et al, 1996). After TW03 cells were successfully infected with EBV, a subpopulation of the infected TW03 cells was subcultured without G418. Parental EBV (-) TW03 and established EBV (+) TW03 cells were found to be negative for mycoplasma by both mycoplasma rRNA assay and Hoechst staining (data not shown). Anchorage-independent cell growth was assessed by plating 100 cells to plates $(\phi 3.5 \mathrm{~cm})$ with $0.3 \%$ agarose/IMDM/1\% FCS. Morphological change in cell suspension cultures was also examined by plating cells in chamber slides treated by Sigmacote (Sigma, St. Louis, Missouri) as reported previously (Knox et al, 1996). The size of the colonies was analyzed using NIH Image 1.6 software (National Institutes of Health, Bethesda, Maryland) after capturing the images $(567 \times 443$ pixels $)$ at $\times 25$ magnification with a CCD camera, HC-2000 3CCD (Fujifilm, Tokyo, Japan). Cell proliferation was estimated by direct counting of the cells in flasks after staining with $0.2 \%$ trypan blue.

\section{EBV Infection of TW03 Cells}

Semi-confluent EBV (-) TW03 cells were cocultured for 2 days with the neo-Akata cells irradiated at 15,000 rad. Then the floating cells and cell debris were removed by 2 to 3 washings with phosphate buffered saline (PBS). The infected cells were cultured in IMDM supplemented 

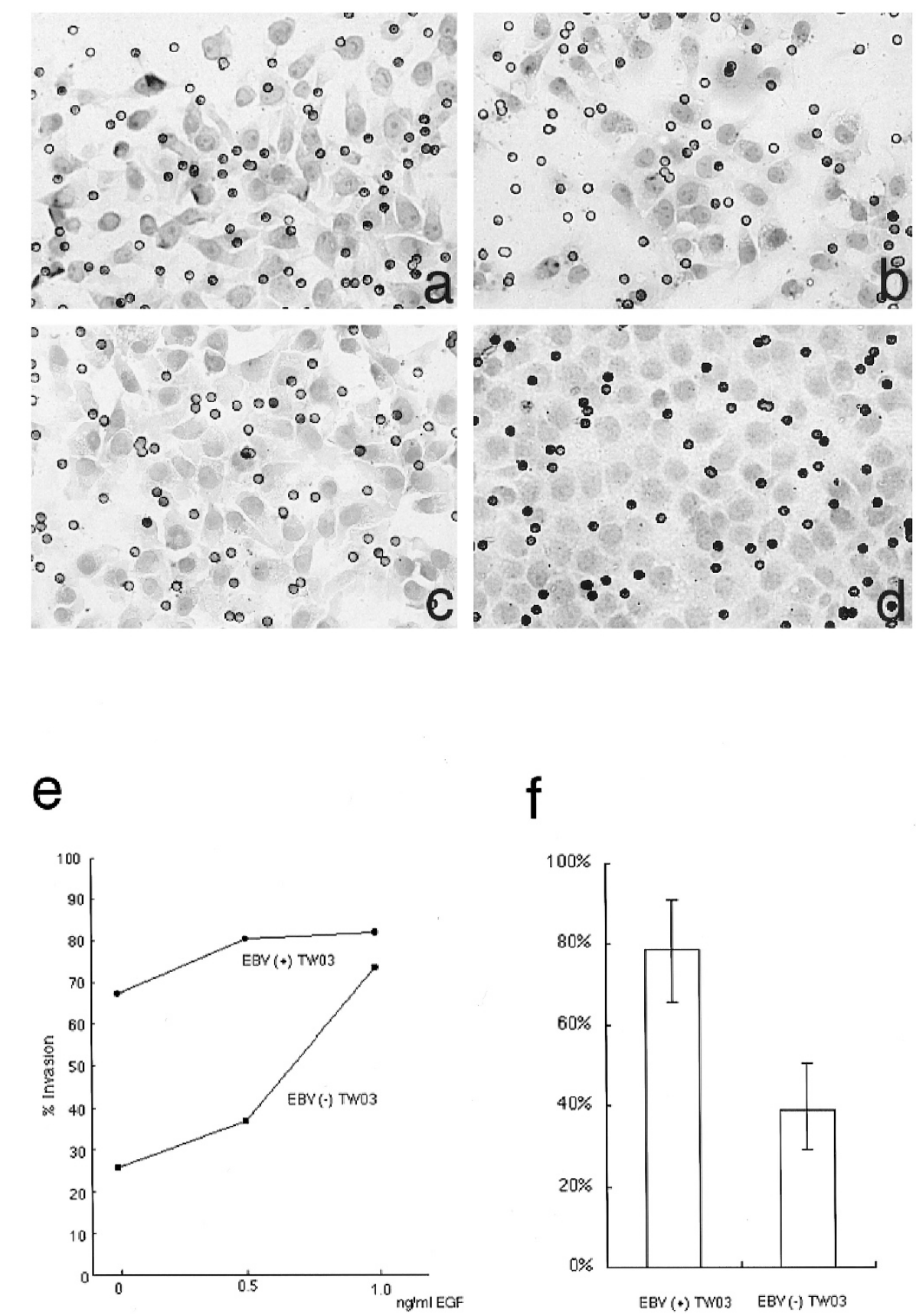

\section{Figure 5.}

Invasion assay. $a, c$ : EBV (+) TW03; $b, d$; EBV (-) TW03; $a, b$ : a GFR-Matrigel invasion chamber; $c$, $d$ : a control chamber. Note that both EBV $(+)$ and EBV $(-)$ TW03 cells show invasive activity. However the EBV $(+)$ TW03 culture had more invaded cells. Small circles are $8.0 \mu \mathrm{m}$ pores. Epidermal growth factor (EGF; 0.5 $\mathrm{ng} / \mathrm{ml}$ ) were added in the culture. Hematoxylin staining. Original magnification, $\times 200$. $e$ : The percent of invasion at different EGF concentrations. A preliminary study. $f$, The percent of invasion of EBV $(+)$ and $(-)$ TW03 cells at a concentration of $0.5 \mathrm{ng} / \mathrm{ml}$ EGF.

with $10 \%$ FCS and G418 $(200 \mu \mathrm{g} / \mathrm{ml})$. The culture medium was exchanged once or twice weekly.

\section{In Situ Hybridization and Immunostaining}

EBERISH was performed as reported previously (Teramoto et al, 1996). Deparaffinized sections were treated with $0.2 \mathrm{~N} \mathrm{HCl}$ for 10 minutes and then with 5 $\mu \mathrm{g} / \mathrm{ml}$ proteinase $\mathrm{K}$ in $50 \mathrm{~mm}$ Tris- $\mathrm{HCl}(\mathrm{pH} 8.0)$ at $37^{\circ} \mathrm{C}$ for 15 minutes. In situ hybridization was performed by incubating sections with $0.5 \mathrm{ng} / \mu \mathrm{l}$ of FITC-labeled antisense EBER-1 oligonucleotide probe at $37^{\circ} \mathrm{C}$ overnight. As a control probe, a GC-content-matched oligonucleotide labeled with FITC was used. Detection of the reaction products was performed with an in situ detection kit (K046; Dakopatts, Glustop, Denmark). Positive signals were visualized as dark blue or black staining on nuclei with BCIP/NBT. In situ hybridization for histone mRNA was performed using a commercial probe (Dakopatts) according to the supplier's instruc- 

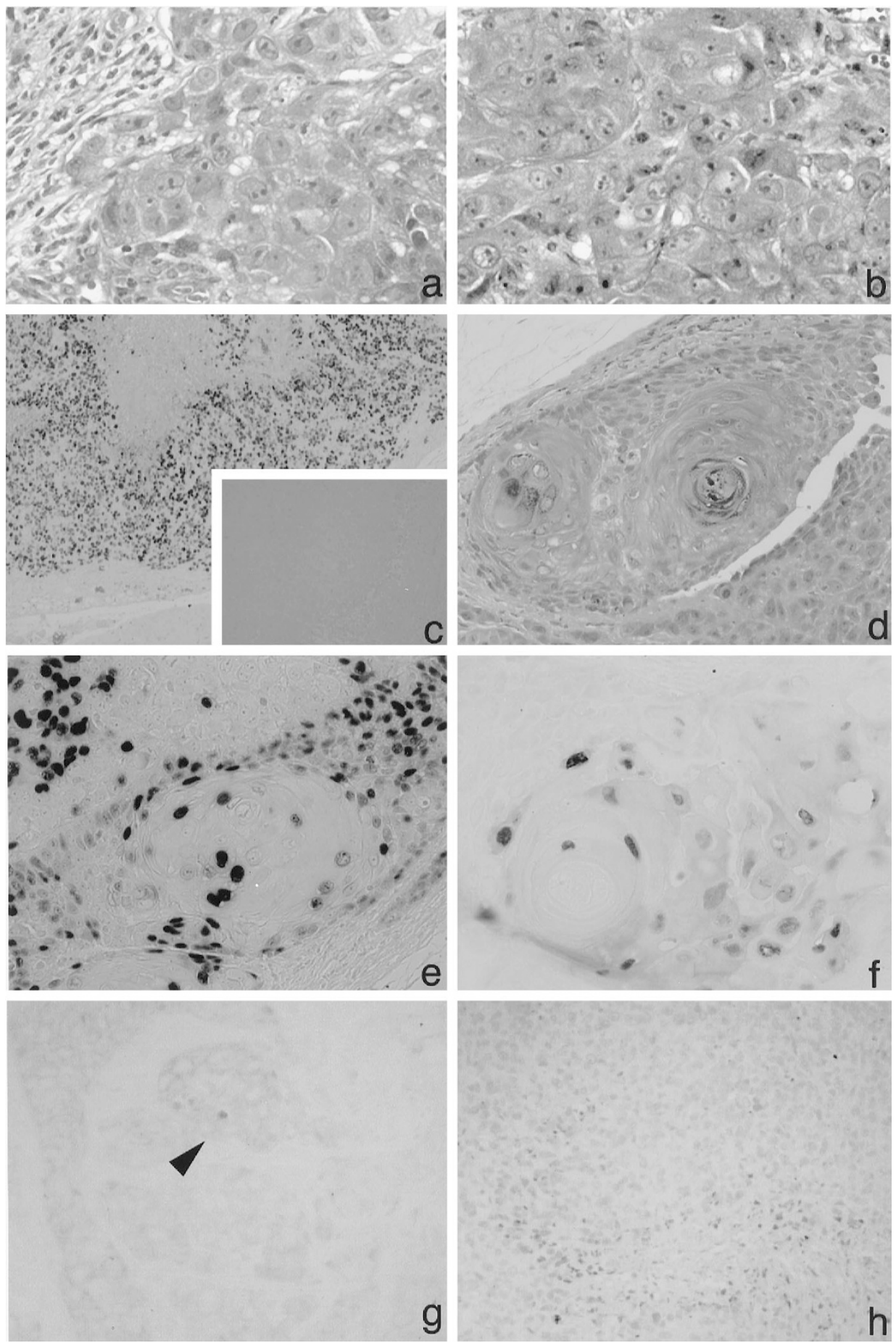

\section{Figure 6.}

Histology of nude mouse tumors and SCID mouse tumors. $a$, an EBV (+) TW03-induced SCID tumor. HE staining. $b$, an EBV (-) TW03-induced SCID tumor. HE staining. $c$, EBER-1 in situ hybridization of the EBV (+) TW03-induced nude mouse tumor. EBER-1 was visualized as BCIP/NBT deposition on nuclei. Inset: control in situ hybridization with $d$ : a horny pearl in the EBV $(+)$ TW03-induced SCID mouse tumor. HE staining. e, EBER-1 in situ hybridization of the horny pearl in the EBV $(+)$ TW03-induced SCID mouse tumor. $f$, Immunostaining against ZEBRA for the same horny pearl. $g$, Immunostaining against ZEBRA. A ZEBRA positive cell is arrowed. $h$, Immunostaining against LMP1. Original magnification: $\times 25(c), \times 200(a, b, d$ to $f), \times 100(g, h)$.

tions. Immunohistochemical staining was performed by the conventional avidin-biotin complex method. An antigen retrieval procedure was performed if necessary. Simultaneous immunostaining against Ki-67 or cytokeratin and EBERISH was performed as described previously (Teramoto et al, 1996). Staining with EBERISH was performed first and then the specimens were subjected to immunostaining. The monoclonal antibodies and antisera used in this study are listed in Table 1. Anticomplement immunofluorescence (ACIF) staining with human monospecific sera for EBNA1 was performed as described previously (Jian et al, 1991). 

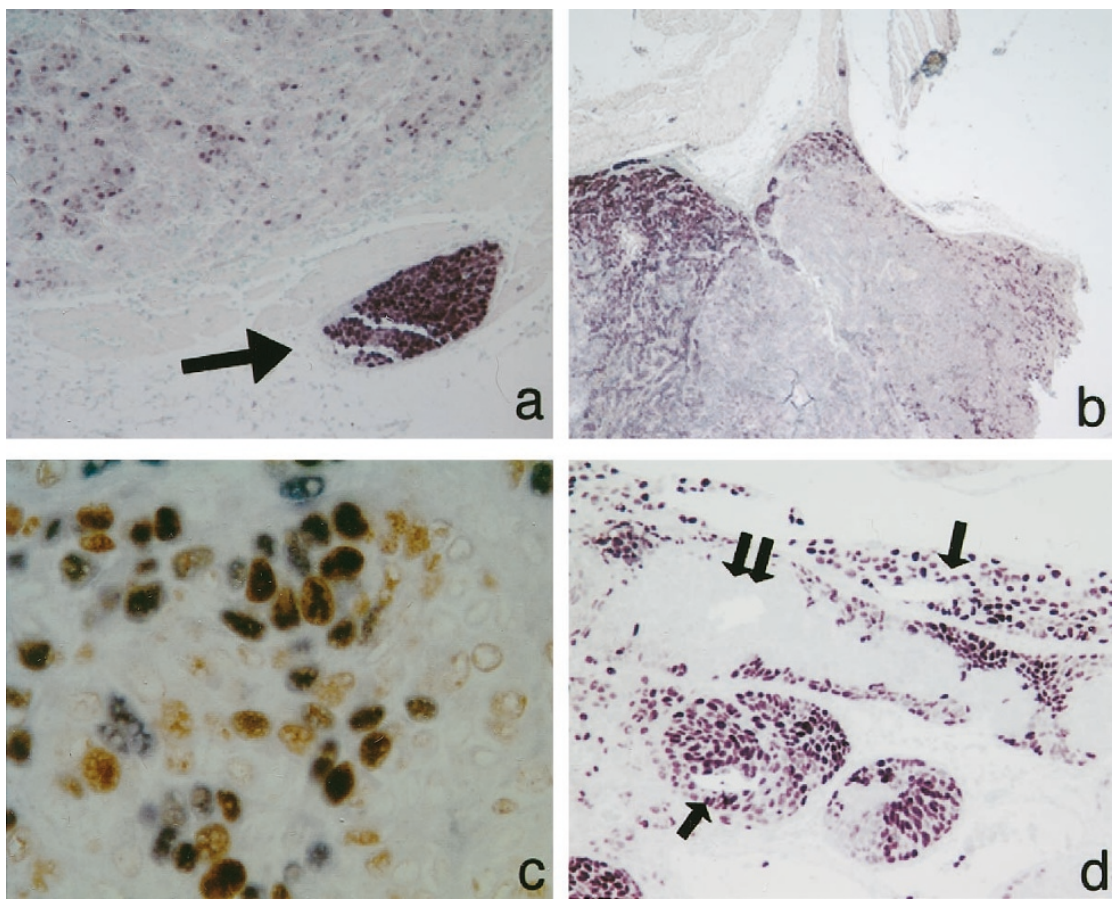

\section{Figure 7.}

Distribution of EBV-positive TW03 cells in EBV (+) TW03-induced SCID mouse tumors detected by EBER-1 in situ hybridization. a, Note that EBER-1 positive cells were less frequent in the main tumor (left upper part), but virtually all cells were EBER-1 positive in the daughter nodule (arrow). $b$, EBER-1 positive cells are found more frequently in the periphery of the main tumor. $c$, Simultaneous staining for Ki67 and EBER-1. Ki-67, brown; EBER-1, dark blue. $d$, EBER-1 positive cells (arrow) and negative cells (double arrow) were microdissected and were subjected to PCR. Original magnification: $\times 100(a$ and $d), \times 10(b), \times 400(c)$.

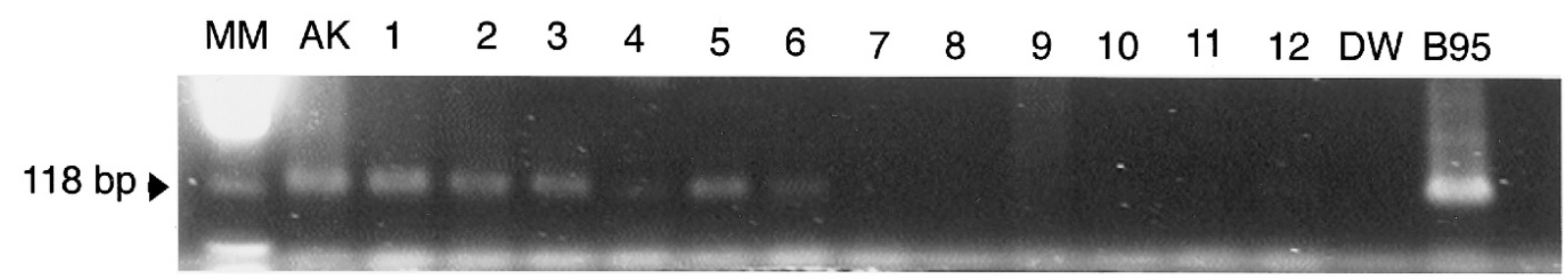

Figure 8.

Microdissection PCR. PCR products (125 bp) from BamHIW fragment (arrowhead) were amplified from neo-Akata cells (Ak), B95-8 (B95) cells and EBER-1 positive tissue fragments (lanes 1-6), but not from EBER-1 negative tissue fragments (lanes 7-12) or distilled water (DW).

Table 1. The Antibodies

\begin{tabular}{llll}
\hline Specificity & Species & \multicolumn{1}{c}{ Clone } & \multicolumn{1}{c}{ Reference } \\
\hline EBNA1 & human & & Jian WQ et al. \\
EBNA2 & Mmab & PE2 & Dakopatts \\
LMP1 & Mmab & CS14 & Dakopatts \\
ZEBRA & Mmab & BZ-1 & Dakopatts \\
EA-D & Mmab & EADE31 & Novocastra \\
cytokeratin & Mmab & AE1, AE3 & Dakopatts \\
Ki67 & Mmab & Ki67 & Dakopatts \\
HLA-DR & Mmab & LN-3 & Novocastra \\
Bcl-2 & Mmab & 124 & Dakopatts \\
\hline
\end{tabular}

Mmab, mouse monoclonal antibody.

\section{Polymerase Chain Reaction}

The EBV genome was amplified using the PCR primers for the BamHIW fragments, TC60 and TC61 (Saito et al, 1989). RT-PCR was performed by slight modifi- cation of the method of Chen et al (1995), using the primers for $Q$ promoter-generated transcripts (QUK EBNA1: outer primers, 5'-AGCGTGCGCTACCGGAT and 5'-CAATGCAACTTGGACGTTIT; inner primers, 5'-GTGCGCTACCGGATGGCG and 5'-CATTTCCAG GTCCTGTACCT) and for $\mathrm{C}$ or W promoter-generated transcripts (YUK EBNA1: outer primers, 5'-CGTGTG ACGTGGTGTAAAGT and 5'-CAATGCAACTTGGACG TTT; inner primers, 5'-CGTGTGACGTGGTGTAAAGT and 5'-CATTTCCAGGTCCTGTACCT). PCR products were separated in $2 \%$ NuSieve gel and stained with $0.1 \%$ ethidium bromide.

PCR of microdissected tissue specimens was performed as described previously (Teramoto et al, 1992). The cover glass was removed from the specimens stained with EBERISH. Parts of tissue sections, either positive or negative for EBER-1, were microdissected using the glass tip of a micromanipulator. The dissected specimens were digested with proteinase 
$\mathrm{K} / \mathrm{Tris}-\mathrm{HCl}, 10 \mathrm{~mm}, \mathrm{pH}$ 8.0/0.1\% NP-40 and subjected to 53 cycles of amplification of the BamHIW fragment of EBV.

\section{Southern Blot Hybridization}

The extracted DNA was digested with BamHI or with BamHI and Bg/ll (Bethesda Research Laboratories, Rockville, Maryland). After transfer onto nylon membranes, the digested DNA was hybridized with a $1.9 \mathrm{~kb}$ Xhol fragment of EBV DNA (containing the sequence adjacent to the EBV terminal repeat) (Anagnostopoulos et al, 1989). The hybridized probe was detected using Gene Images (Amersham Co., Ltd., Tokyo, Japan).

\section{Transplantation into SCID and Nude Mice}

EBV (-) TW03 and EBV (+) TW03 cells were injected subcutaneously into SCID mice (C.B-17/lcr Crj-scid, Charles River Japan Inc., Yokohama, Japan) and nude mice (Crj:CD-1 (ICR)-nu/nu, Charles River Japan Inc.); $5 \times 10^{6}$ cells to SCID mice and $5 \times 10^{4}, 5 \times 10^{5}$, and $5 \times 10^{6}$ cells to nude mice. The mice were autopsied, and the tissue specimens were subjected to histopathological examination.

\section{Invasion Assay}

An invasion assay was performed using BIOCOAT growth-factor-reduced Matrigel (GFR Matrigel) invasion chambers (Becton Dickinson, Bedford, Massachusetts) according to the supplier's instructions. Briefly, $5 \times 10^{4}$ cells were plated into a culture insert containing IMDM/0.1\% FCS supplemented with an epidermal growth factor (EGF) (Boehringer Manheim Scandinavia, Bromma, Sweden). The cells with an invasive ability passed from the inside to the outside of the wells through a GFR Matrigel matrix, a reconstituted basement membrane in vitro. After removing all of the remaining cells from the inside of the inserts, the invaded cells were counted on photographs taken at $\times 200$ magnification. Control inserts were not sealed with a GFR Matrigel matrix. The invasion index was $100 \times$ the number of cells invaded in the GFR Matrigel matrix divided by the number of cells invaded in the control.

\section{Acknowledgement}

We thank the following for their skillful technical assistance: Karin Kvarnung, Sachiyo Onoda, Mutsumi Okabe, Reiko Endo, Yoshie Sakamoto, Hiromi Nakamura, Rika Hayashi, and Miyuki Shiotani. T. N. and M. A. are recipients of a fellowship from the Cancer Research Institute in New York.

\section{References}

Anagnostopoulos I, Herbst H, Niedobitek G, and Stein H (1989). Demonstration of monoclonal EBV genomes in Hodgkin's disease and KI-1-positive anaplastic large cell lymphoma by combined Southern blot and in situ hybridization. Blood 74:810-816.
Chen F, Zou JZ, di Renzo L, Winberg G, Hu LF, Klein E, Klein G, and Ernberg I (1995). A subpopulation of normal B cells latently infected with Epstein-Barr virus resembles Burkitt lymphoma cells in expressing EBNA-1 but not EBNA-2 or LMP1. J Virol 69:3752-3758.

Holmgren L, Szeles L, Rajnavolgyi E, Folkman J, Klein G, and Falk KI (1999). Horizontal transfer of Epstein-Barr virus DNA by uptake of apoptotic bodies. Blood 93:3956-3963.

Imai S, Nishikawa J, and Takada K (1998). Cell-to-cell contact as an efficient mode of Epstein-Barr virus infection of diverse human epithelial cells. J Virol 72:4371-4378.

Jian WQ, Wendel-Hansen V, Lundkvist A, Ringertz N, Klein G, and Rosen A (1991). Intranuclear distribution of EpsteinBarr virus-encoded nuclear antigens EBNA-1,-2,-3, and -5 . J Cell Sci 99:497-502.

Kieff E (1996). Epstein-Barr virus and its replication. In: Fields BN, Knipe DM, and Howley PM, eds. Fields Virology. Philadelphia, PA: Lippincott-Raven, 2343-2396.

Klein G (1994). Epstein-Barr virus strategy in normal and neoplastic B cells. Cell 77:791-793.

Knox P, Li Q, Rickinson A, and Young L (1996). In vitro production of stable Epstein-Barr virus-positive epithelial cell clones which resemble the virus:cell interaction observed in nasopharyngeal carcinoma. Virology 215:40-50.

Komano J, Sugiura M, and Takada K (1998). Epstein-Barr virus contributes to the malignant phenotype and to apoptosis resistance in Burkitt's lymphoma cell line Akata. J Virol 72:9150-9156.

Lin CT, Chan W, Chen W, Huang H, Wu H, Hsu M, Chuang S, and Wang $C$ (1993). Characterization of seven newly established nasopharyngeal carcinoma cell lines. Lab Invest 68: 716-727.

Lin CT, Lin CR, Tan GK, Chen W, Dee AN, and Chan WY (1997). The mechanism of Epstein-Barr virus infection in nasopharyngeal carcinoma cells. Am J Pathol 150:17451756.

Niedobitek G, Young LS, Lau R, Brooks L, Greenspan D, Greenspan JS, and Rickinson AB (1991). Epstein-Barr virus infection in oral hairy leukoplakia: Virus replication in the absence of a detectable latent phase. J Gen Virol 72:30353046.

Nishikawa J, Imai S, Oda T, Kojima T, Okita K, and Takada K (1999). Epstein-Barr virus promotes epithelial cell growth in the absence of EBNA2 and LMP1 expression. J Virol 73: 1286-1292.

Pathmanathan R, Prasad U, Chandrika G, Sadler R, Flynn K, and Raab-Traub N (1995). Undifferentiated, nonkeratinizing, and squamous cell carcinoma of the nasopharynx. Variants of Epstein-Barr virus-infected neoplasia. Am J Pathol 146: 1355-1367.

Raab TN (1992). Epstein-Barr virus and nasopharyngeal carcinoma. Semin Cancer Biol 3:297-307.

Rickinson AB and Kieff E (1996). Epstein-Barr virus. In: Fields BN, Knipe DM, and Howley PM, eds. Fields Virology. Philadelphia, PA: Lippincott-Raven, 2397-2446.

Roth G, Curiel T, and Lacy J (1994). Epstein-Barr viral nuclear antigen 1 antisense oligodeoxynucleotides inhibits proliferation of Epstein-Barr virus-immortalized B cells. Blood 84: 582-587. 
Rowe M, Lear AL, Croom CD, Davies AH, and Rickinson AB (1992). Three pathways of Epstein-Barr virus gene activation from EBNA1-positive latency in B lymphocytes. J Virol 66: 122-131.

Saito I, Servenius B, Compton T, and Fox RI (1989). Detection of Epstein-Barr virus DNA by polymerase chain reaction in blood and tissue biopsies from patients with Sjogren's syndrome. J Exp Med 169:2191-2198.

Sheu L, Chen A, Meng C, Ho K, Lee W, Leu F, and Chao C (1996). Enhanced malignant progression of nasopharyngeal carcinoma cells mediated by the expression of Epstein-Barr nuclear antigen 1 in vivo. J Pathol 180:243-248.

Shimizu N, Yoshiyama H, and Takada K (1996). Clonal propagation of Epstein-Barr virus (EBV) recombinants in EBV-negative Akata cells. J Virol 70:7260-7263.

Teramoto N, Akagi T, Yoshino T, Takahashi K, and Jong H (1992). Direct detection of Epstein-Barr Virus DNA from a single Reed-Sternberg cell of Hodgkin's disease by polymerase chain reaction. Jpn J Cancer Res 83:329-333.
Teramoto N, Sarker AB, Tonoyama Y, Yoshino T, Hayashi K, Takahashi K, and Akagi T (1996). Epstein-Barr virus infection in the neoplastic and nonneoplastic cells of lymphoid malignancies. Cancer 77:2339-2347.

Trivedi P, Masucci M, Winberg G, and Klein G (1991). The Epstein-Barr-virus-encoded membrane protein LMP but not the nuclear antigen EBNA-1 induces rejection of transfected murine mammary carcinoma cells. Int J Cancer 48:794-800.

Wang D, Liebowitz D, and Kieff E (1985). An EBV membrane protein expressed in immortalized lymphocytes transforms established rodent cells. Cell 43:831-840.

Yoshiyama H, Imai S, Shimizu N, and Takada K (1997). Epstein-Barr virus infection of human gastric carcinoma cells: implication of the existence of a new virus receptor different from CD21. J Virol 71:5688-5691. 